\title{
Hemodynamic instability associated with dependent lung collapse in lateral position with kidney rest during laparoscopic nephroureterectomy
}

\author{
Tae-Young Kim, Seongwook Jeong, Seongheon Lee \\ Department of Anesthesiology and Pain Medicine, Chonnam National University Medical School, Chonnam National University Hospital, \\ Gwangju, Korea
}

Received September 16, 2020

Revised October 5, 2020

Accepted October 7, 2020

Corresponding author

Seongheon Lee

Department of Anesthesiology

and Pain Medicine, Chonnam

National University Medical School,

Chonnam National University

Hospital, 42 Jebong-ro, Dong-gu,

Gwangju 61469, Korea

Tel: +82-62-220-6895

Fax: +82-62-232-6294

E-mail: aneshead@gmail.com

ORCID:

https://orcid.org/0000-0002-2675-2521
Intraoperative lung collapse is a rarely reported but potentially life-threatening event. We report an unusual case of hemodynamic instability secondary to collapse of the dependent lung. A 76-year-old man was scheduled for right laparoscopic nephroureterectomy. The anesthetized patient was placed in the left lateral position, and the kidney rest beneath iliac crest was elevated. Bilateral breath sounds were confirmed with auscultation. At 40 min after pneumoperitoneum, profound hypotension and high degree atrioventricular block occurred suddenly without desaturation. The operation stopped, and the patient was turned to supine position promptly. Then, blood pressure and electrocardiogram became normal. The chest $x$-ray showed the left lung collapse with proper position of endotracheal tube. We suspect that the tube moved gradually close to or into the main bronchus of nondependent lung after elevation of the kidney rest and/or pneumoperitoneum. Dynamic hyperinflation of nondependent lung might be the cause of hemodynamic instability.

Keywords: Circulatory collapse; Endobronchial intubation; Kidney rest; Pulmonary atelectasis

\section{INTRODUCTION}

Intraoperative lung collapse is a rarely reported but potentially life-threatening event, requiring prompt identification and management [1-3]. A common cause of unilateral lung collapse is an inadvertent endobronchial intubation, which can be occurred during surgical positioning [4,5]. Therefore, thoracic auscultation should be performed carefully not only after endotracheal intubation but also after surgical positioning. Even though bilateral normal breath sounds were auscultated at first, tube migration may occur gradually over time during surgery [6,7]. Nevertheless, unilateral lung collapse can be detected relatively easily by desaturation before serious complications occur. Herein, we reported an unusual case of sudden hemodynamic instability secondary to intraoperative collapse of the dependent lung during laparoscopic urologic surgery in the lateral position with kidney rest. This event occurred without desaturation at about 40 minutes after pneumoperitoneum. 


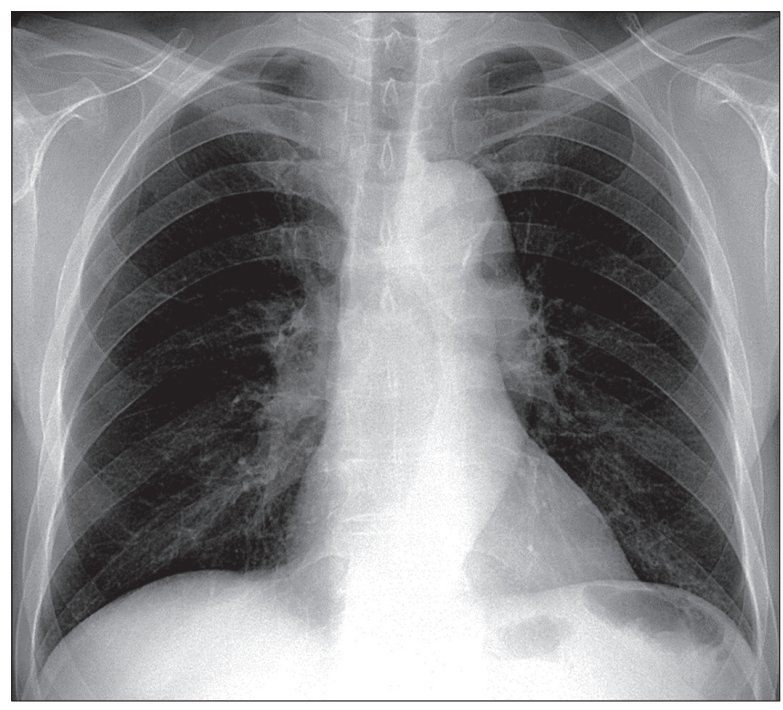

Fig. 1. A normal preoperative chest $\mathrm{x}$-ray (posteroanterior view).

\section{CASE REPORT}

A 76-year-old man (height $162 \mathrm{~cm}$; weight $56 \mathrm{~kg}$ ) was admitted to hospital for management of a renal pelvis tumor in the upper and mid pole of the right kidney and scheduled for right laparoscopic nephroureterectomy under general anesthesia. The patient indicated that he had stopped taking his antihypertensive medications three years ago, and his blood pressures were within normal range during preoperative evaluation. An electrocardiogram showed no abnormal findings with a normal sinus rhythm, and an echocardiogram showed no abnormal findings with ejection fraction of $75 \%$. There were no abnormal findings on physical examinations, laboratory tests, pulmonary function test results, or chest x-ray (Fig. 1). After admission for surgery, his blood pressures were maintained around a systolic blood pressure of 130-160 mmHg.

On arrival in the operating room, his vital signs showed a blood pressure of 194/66 mmHg, a heart rate of 56 beat per minute (bpm), and an oxygen saturation of $100 \%$ on room air. Anesthesia was induced and maintained with propofol and remifentanil using target-controlled infusion system. Tracheal intubation with an $8.0 \mathrm{~mm}$ cuffed endotracheal tube was facilitated with $50 \mathrm{mg}$ of rocuronium. The endotracheal tube was secured at $23 \mathrm{~cm}$ at the lips, and normal breath sounds were heard equally on both sides of the chest. A radial artery cannula was place in the left radial artery for continuous hemodynamic monitoring. The patient was ven- tilated with volume-controlled mode at the following setting: tidal volume of $8 \mathrm{~mL} / \mathrm{kg}$ (predicted body weight), respiratory rate of $12 \mathrm{cycles} / \mathrm{min}$, and positive end expiratory pressure $5 \mathrm{cmH}_{2} \mathrm{O}$ with $50 \%$ of oxygen.

The patient was placed in the left lateral decubitus position for a laparoscopic retroperitoneal approach to the right kidney, and the kidney rest beneath the dependent iliac crest was elevated to allow better exposure of the kidney. There were no remarkable changes in vital signs after position change. Bilateral breath sounds were confirmed again with auscultation to exclude the possibility of endotracheal tube displacement. Before pneumoperitoneum, peak airway pressure was maintained within $20 \mathrm{cmH}_{2} \mathrm{O}$ and end-tidal $\mathrm{CO}_{2}\left(\mathrm{EtCO}_{2}\right)$ was controlled between 31 and $35 \mathrm{mmHg}$. The retroperitoneal space was hydrostatically dilated, and $\mathrm{CO}_{2}$ was insufflated to establish a pneumoperitoneum at a pressure of $12 \mathrm{mmHg}$ about 30 minutes after position change. Peak airway pressure and $\mathrm{EtCO}_{2}$ started to elevate after pneumoperitoneum, and we gradually increased tidal volume to $10 \mathrm{~mL} / \mathrm{kg}$ and respiratory rate to 20 cycles/min to maintain $\mathrm{EtCO}_{2}$ 40-45 mmHg at least.

At about 40 minutes after pneumoperitoneum, peak airway pressure was $30 \mathrm{cmH}_{2} \mathrm{O}$ and $\mathrm{EtCO}_{2}$ was $43 \mathrm{mmHg}$. However, $\mathrm{SpO}_{2}$ remained $100 \%$ with $50 \%$ of oxygen, blood pressure was 115/55 $\mathrm{mmHg}$, and heart rate was $54 \mathrm{bpm}$. The surgical procedure was uneventful until that time, though a sudden hemodynamic instability with bradycardia (35 bpm) and hypotension (95/48 $\mathrm{mmHg}$ ) occurred. The surgical team was informed, and the surgical procedure was stopped. Despite multiple bolus injections of ephedrine (total $28 \mathrm{mg}$ ) and atropine (1 $\mathrm{mg})$, blood pressure decreased to $60 / 30 \mathrm{mmHg}$ and the electrocardiogram converted to a high degree atrioventricular block with a ventricular rate of 20 bpm. A prominent ST-segment depression was also noted in lead II. The pneumoperitoneum pressure was kept below $13 \mathrm{mmHg}$ and $\mathrm{SpO}_{2}$ still remained 99\%. The open surgical wound was rapidly covered with a sterile drape while preparing for epinephrine injection and transcutaneous pacing. Then the patient was turned promptly from lateral to supine position. Immediately after position change, the patient's blood pressure and heart rate started to elevate dramatically without any cardiovascular drugs. The atrioventricular block was converted to normal sinus rhythm (70 bpm) and blood pressure was raised up to $140 / 70 \mathrm{mmHg}$ within 5 minutes. 


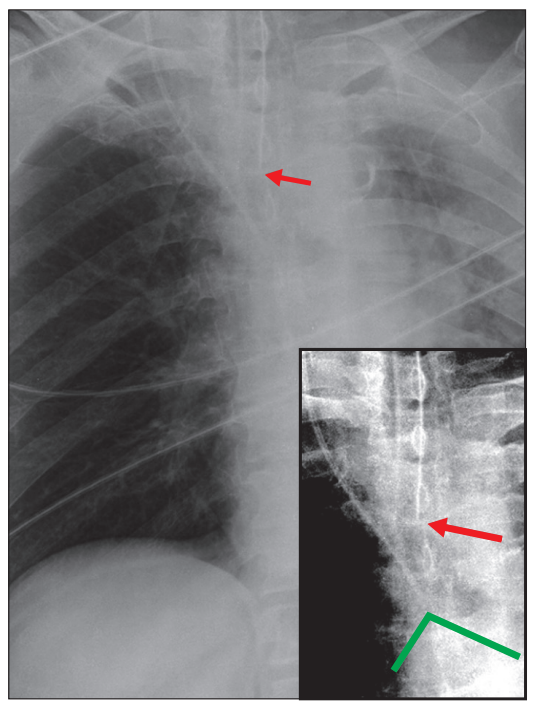

Fig. 2. A chest $x$-ray after position change to supine position (anteroposterior view). It is showing that the left lung is collapsed, and the endotracheal tube tip (red arrow) is placed at $2.9 \mathrm{~cm}$ above the carina (green).

Chest auscultation revealed absent breath sounds in the left hemithorax. Arterial blood gas analysis and an anteroposterior chest $\mathrm{x}$-ray were subsequently performed at 10 minutes after position change. Arterial blood gas analysis demonstrated $\mathrm{PaO}_{2}$ of $137 \mathrm{mmHg}$ with $\mathrm{FiO}_{2} 0.52$ and $\mathrm{PaCO}_{2}$ of $58 \mathrm{mmHg}$ with $\mathrm{pH}$ of 7.28 , while $\mathrm{EtCO}_{2}$ was $33 \mathrm{mmHg}$ in the supine position. The chest $\mathrm{x}$-ray showed the left lung collapse (Fig. 2). The tip of endotracheal tube was in proper position at about $2.9 \mathrm{~cm}$ far from the carina.

We tried to recruit the left lung via squeezing a manual respiratory bag carefully, and then applied the positive end-expiratory pressure of $7 \mathrm{mmHg}$. Re-expansion of left lung was confirmed by auscultation. With intravenous nitroglycerin infusion, the ST-segment depression resolved over 30 minutes. About 1 hour after position change to supine, arterial blood gas analysis showed almost normal $\mathrm{pH} 7.34$, $\mathrm{PaO}_{2} 123 \mathrm{mmHg}, \mathrm{PaCO}_{2} 46 \mathrm{mmHg}$ with $\mathrm{FiO}_{2}$ 0.54. The operation was completed by open laparotomy in semi-lateral position. After operation, the patient was transferred to an intensive care unit for observation. A follow-up chest $\mathrm{x}$-ray after surgery showed the complete remission of collapse (Fig. 3). He discharged from hospital 5 days after operation without any problem.

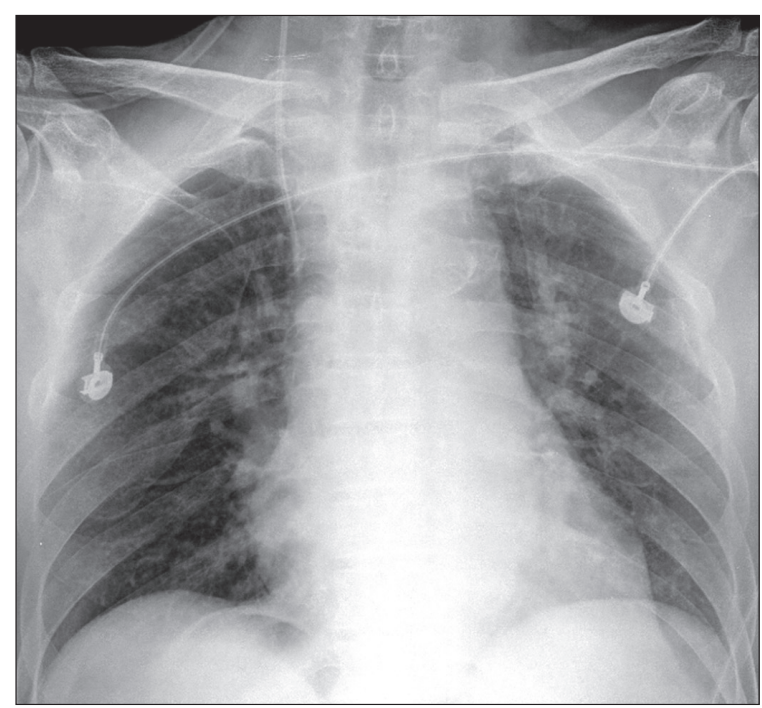

Fig. 3. A follow-up chest x-ray after surgery (anteroposterior view). It is showing that the left lung is fully expanded.

\section{DISCUSSION}

This case report demonstrates an example of intraoperative collapse of the dependent lung during laparoscopic urologic surgery in the lateral position with kidney rest that caused profound hypotension and bradycardia. Intraoperative lung collapse has been reported previously, however, few involved hemodynamic instability without hypoxemia.

The mechanism of lung collapse in this patient is unclear. Most common cause of intraoperative unilateral lung collapse is an inadvertent endobronchial intubation. In this case, the initial depth of endotracheal tube might be proper because chest $\mathrm{x}$-ray checked in supine position revealed that the tip of the endotracheal tube was placed $2.9 \mathrm{~cm}$ above the carina (Fig. 2). Bilateral normal breath sounds were also confirmed on auscultation after intubation and after position change to lateral decubitus. Therefore, endobronchial displacement of endotracheal tube during surgical positioning might be ruled out.

However, it cannot be excluded that endotracheal tube migration occurred gradually over time during surgery [G]. Exposure of the kidney was facilitated by elevating the kidney rest beneath the dependent iliac crest in a lateral decubitus position, and excessively elevated kidney rest might cause shortness of distance from the tip of endotracheal tube to carina. Since the upper body was placed as in the Trendelenburg position and abdomen was insufflated 
with $\mathrm{CO}_{2}$ for pneumoperitoneum, the tip of endotracheal tube can become more closer to the carina [7-9]. Based on these considerations, we suspect that the endotracheal tube moved close to or into right main bronchus at some time point after the kidney rest elevation and/or pneumoperitoneum.

Another common cause of lung collapse is a bronchus obstruction by mucus plugs or blood clots. However, it may be excluded in this case because there was no prominent secretion or blood when suctioning. Furthermore, just repositioning the patient into the supine position improved vital signs before suctioning.

We suspect that the nondependent lung was hyperinflated while the dependent lung collapsed in the present case, similarly to other cases of endobronchial intubation. Also, development of hyperinflated lung is associated not only with a high degree of airway obstruction but also short expiratory time and a large tidal volume [10]. Due to increasing $\mathrm{EtCO}_{2}$ during pneumoperitoneum, the patient was hyperventilated by increasing the respiratory rate to 20 cycles/min and the tidal volume to $10 \mathrm{~mL} / \mathrm{kg}$. From a cardiovascular perspective, lung hyperinflation can increase intrathoracic pressure, pulmonary vascular resistance, and right ventricular afterload. This can result in decreased venous return, left ventricular compliance, and contractility [11,12]. Therefore, we think that mechanical pressure of the hyperinflated lung might be the cause of hemodynamic instability in this patient just as seen in a tension pneumothorax, rather than hypoxia-induced bradycardia and hypotension. Desaturation was not observed even while sudden bradycardia and hypotension sustained for several minutes in the present case.

In conclusion, we suspect that the endotracheal tube moved gradually close to or into the main bronchus of nondependent lung after elevation of the kidney rest and/or pneumoperitoneum. Dynamic hyperinflation of nondependent lung might be the cause of hemodynamic instability. We suggest that the use of the kidney rest during laparoscopic surgery in lateral position can cause the endotracheal tube migration.

\section{CONFLICT OF INTEREST}

No potential conflict of interest relevant to this article was reported.

\section{REFERENCES}

1. Pivalizza EG, Tonnesen AS. Acute life-threatening intraoperative atelectasis. Can J Anaesth 1994;41:857-60.

2. Samuels SI, Brodsky JB. Profound intraoperative atelectasis. Br J Anaesth 1989;62:216-8.

3. Hedenstierna G, Edmark L. Mechanisms of atelectasis in the perioperative period. Best Pract Res Clin Anaesthesiol 2010;24:157-69.

4. Mackenzie M, MacLeod K. Repeated inadvertent endobronchial intubation during laparoscopy. Br J Anaesth 2003;91: 297-8.

5. Oh S, Chung JH, Lee SM, Chung KD, Cho HS, Han YJ, et al. Delayed hypoxemia due to endobronchial intubation as a result of positioning the patient under general anesthesia: a case report. Anesth Pain Med 2011;6:63-6.

6. Nishikawa K, Nagashima C, Shimodate Y, Igarashi M, Namiki A. Migration of the endotracheal tube during laparoscopy-assisted abdominal surgery in young and elderly patients. Can J Anaesth 2004;51:1053-4.

7. Gupta N, Girdhar KK, Misra A, Anand R, Kumar A, Gunjan. Tube migration during laparoscopic gynecological surgery. J Anaesthesiol Clin Pharmacol 2010;26:537-8.

8. Lobato EB, Paige GB, Brown MM, Bennett B, Davis JD. Pneumoperitoneum as a risk factor for endobronchial intubation during laparoscopic gynecologic surgery. Anesth Analg 1998;86:301-3.

9. Kim JH, Hong DM, Oh AY, Han SH. Tracheal shortening during laparoscopic gynecologic surgery. Acta Anaesthesiol Scand 2007;51:235-8.

10. Tan IK, Bhatt SB, Tam YH, Oh TE. Effects of PEEP on dynamic hyperinflation in patients with airflow limitation. $\mathrm{Br} \mathrm{J}$ Anaesth 1993;70:267-72.

11. Conacher ID. Dynamic hyperinflation--the anaesthetist applying a tourniquet to the right heart. Br J Anaesth 1998; 81:116-7.

12. Myles PS, Madder H, Morgan EB. Intraoperative cardiac arrest after unrecognized dynamic hyperinflation. $\mathrm{Br} \mathrm{J}$ Anaesth 1995;74:340-2. 